\title{
Adaptive Nonlinear Tracking Control of an Underactuated Nonminimum Phase Model of a Marine Vehicle Using Ultimate Boundedness
}

\author{
Yannick Morel \\ Department of Ocean Engineering \\ Florida Atlantic University - Sea Tech \\ Dania Beach, FL 33004-3023 \\ ymorelaseatech. fau. edu
}

\author{
Alexander Leonessa \\ Department of Mechanical, Materials and \\ Aerospace Engineering \\ University of Central Florida \\ Orlando, FL 32816-2450 \\ aleo@mail.ucf.edu
}

\begin{abstract}
In this paper, an adaptive nonlinear tracking controller for an underactuated nonminimum phase model of a marine vehicle is derived. The result is kept flexible enough, throughout its derivation, to be applicable to a large variety of marine vehicles. The characteristics of the dynamic model are such that solving the tracking problem is non-trivial. Specifically, we consider a propulsion system composed of either a thruster and a rudder, or a vectored thruster, which provides two independent control commands and three degrees of freedom, with an overall unstable zero-dynamics. The tracking problem dealt with in this paper is solved using a backstepping approach, as well as a technique derived from dynamic surface control theory and the notion of ultimate boundedness. The tracking problem is first solved assuming full knowledge of the geometric and hydrodynamic coefficients appearing in the vehicle's model. The control law is then modified into an adaptive one. Computer simulations are presented to illustrate the performances of the final control algorithm.
\end{abstract}

\section{INTRODUCTION}

The controller derived in this paper is designed for the most commonly used propulsion system available on autonomous underwater vehicles as well as surface vessels: a thruster, used for propulsion, and a rudder for stirring, or, equivalently, a vectored thruster ([1]). The propulsion system considered provides two independent control commands, while the vehicle has three degrees of freedom. The vehicle will thus have fewer independent actuators than degrees of freedom, making it underactuated. The corresponding dynamic model is characterized by an unstable zero-dynamics, as mentioned in [2].

An additional challenge is that the constant parameters characterizing the mathematical model on which the control law is based on are difficult to estimate. Designing a control law not dependent on those parameters is thus highly desirable.

The design of trajectory tracking controllers for underactuated marine vehicles has generated a growing interest over the past few years. Many controllers dealing with such a problem are available in the literature ([2]-[6]). In [3], the case of a surface ship equipped with a pair of propellers is considered. The derived controller yields good results and has the invaluable advantage to be simple enough to implement. However, the desired trajectory is limited to straight lines and circles. In [4], still considering the same type of underactuation as in [3], the authors derive a controller that uses a state estimator to handle uncertainties on state measurement.

In [5], a similar twin propellers scenario is considered. The author, relying on a separation principle, manages to compensate for ocean currents.
Finally, in [2], a controller for an underactuated AUV equipped with a propeller and a side thruster is designed. The corresponding vehicle's model is fairly similar to the one we consider, since it involves a coupling between sway force and yawing moment. The controller handles constant and slow varying external perturbations. However, the system's zero dynamics is stable, while the system we consider has an unstable zero dynamics.

To compensate for the lack of knowledge of a vehicle's mathematical model's constant parameters, one can use an adaptive approach. The design of adaptive controllers for marine vehicles has been widely studied. In [7], the authors present an adaptive control strategy that uses an error variable $\beta$, which corresponds to the angle between the vehicle's longitudinal axis, and the direction of the vehicle's desired position. The resulting controller solves the regulation problem for an underactuated AUV. The controller presented in Section $\mathrm{V}$ builds upon the approach introduced in [7], using the same error variable $\beta$, but extending the problem to trajectory tracking, and dealing with an unstable zero dynamics.

Amplitude saturation effects have been dealt with in [8], where an adaptive control law that enforces actuators saturation by modifying the reference system that the vehicle is tracking is designed. In [9], both actuators amplitude and rate saturations are treated. Specifically, the control algorithm modifies the reference input controlling the motion of the reference system in such a way that the actual vehicle, given its actuators limits, can track the reference system without violating the saturation constraints.

The control of underactuated marine vehicles with an unstable zero dynamics (or underactuated nonminimum phase marine vehicles) is an issue rarely addressed. Only one such controller is available in the literature [10]. But the resulting control law is fairly complex, which makes it difficult to implement.

In this paper, we will introduce a new nonlinear adaptive motion controller for a marine vehicle equipped with either a thruster and a rudder, or a vectored thruster. The design of controllers for underactuated marine vehicles is a problem dealt with in many research papers. However, the assumptions taken in the design of the control algorithms available in the literature are incompatible with the type of propulsion system that we consider. Namely, in our case, two control inputs are used, surge and sway forces, the sway force being used to generate a yawing moment. Most controllers (see for example [3]-[6]) do not account for this sway force, consequently, they can not be expected to provide good performance if implemented on an actual vehicle using a propeller and a rudder, or a vectored thruster. In [2], the authors take into account such a coupling, however, 
their dynamic model does not allow to consider the type of propulsion we target. Indeed, the controller presented in [2], when applied to a vehicle with a side thruster placed behind the vehicle's center of gravity, gives good position tracking, but leads the vehicle to turn around and move backward, which is an unacceptable behavior.

We chose to track a given trajectory in terms of both position and attitude. However, having three degrees of freedom and only two control inputs, it is unrealistic to expect to be able to span all trajectories in the configuration space (surge, sway and yaw). Furthermore, as mentioned in [11], perfect tracking for a nonminimum phase system is not achievable. Accordingly, the control design objective for such a system should not be perfect tracking, but bounded-error tracking. We are able to solve this problem by designing a control strategy that orients the vehicle toward the desired position, and keeps it at a constant distance from it. By doing so, we introduce a constant position error corresponding to the distance between the vehicle and its desired position, which allows to avoid the occurrence of unstable behaviors of the vehicle. By introducing an error over which we have a certain amount of control, we are able to overcome an undesirable behavior which we would not otherwise be able to avoid. To derive such a control algorithm, we use a backstepping technique similar to that introduced in [12].

Being able to implement a controller on a vehicle without having to tune it is a desirable feature. The tuning process can indeed be time consuming and quite demanding. Furthermore, the controller will only perform as well as the tuning allows. Thus, instead of tuning the unknown parameters of the vehicle's model, we take initial estimates which will then be refreshed accordingly to update laws that guarantee good tracking properties. We use a Model-Reference Adaptive Control (MRAC) framework, similar to the one introduced in [9], where the reference model corresponds to a virtual vehicle whose dynamics is described by two independent second order oscillators. This reference system is easily controlled and will accurately track the desired trajectory, while the real system is going to track the reference one.

The stability of the closed loop will be studied using the notion of ultimate boundedness ([13]). Furthermore, we will use a technique derived from dynamic surface control ([14]) to simplify the expression of the control command.

This paper is organized as follows, Section II details the kinematics of the system, Section III describes the vehicle's dynamic model, and Section IV introduces the tracking errors considered. Section V contains the step by step derivation of the control law, assuming perfect knowledge of the vehicle's geometric and hydrodynamic parameters. In Section VI, we transform the obtained control law into an adaptive one. We then present simulation results in Section VII, and our final conclusions in Section VIII.

\section{MARine Vehicle Kinematics}

This paper deals with the design of a controller that only takes into account movements in the horizontal plane. Accordingly, we will use a reduced version of the six degrees of freedom marine vehicle mathematical model introduced in [15], considering only three degrees of freedom.

In three degrees of freedom, the position and attitude of a marine vehicle are described by the vector

$$
\eta \triangleq\left[\begin{array}{lll}
x & y & \psi
\end{array}\right]^{\mathrm{T}}
$$

where $(x, y)$ denotes the position of the vehicle in a two dimensional earth fixed frame of reference ([15]), and $\psi$ is its heading angle. The linear body-fixed velocities are introduced with the following notation,

$$
v \triangleq\left[\begin{array}{lll}
u & v & r
\end{array}\right]^{\mathrm{T}},
$$

where $u, v$ are the forward and lateral velocities, and $r$ is the angular velocity in yaw.

In three degrees of freedom, the projection of a vector expressed in the body-fixed frame ([15]) into the inertial frame is classically accomplished using the transformation matrix

$$
J(\psi) \triangleq\left[\begin{array}{ccc}
\cos (\psi) & -\sin (\psi) & 0 \\
\sin (\psi) & \cos (\psi) & 0 \\
0 & 0 & 1
\end{array}\right]
$$

The opposite operation, which consists of projecting a vector from the inertial frame into the body-fixed frame, is accomplished using $J^{-1}(\psi)$,

$$
J^{-1}(\psi)=\left[\begin{array}{ccc}
\cos (\psi) & \sin (\psi) & 0 \\
-\sin (\psi) & \cos (\psi) & 0 \\
0 & 0 & 1
\end{array}\right]=J^{\mathrm{T}}(\psi) .
$$

Using (1), (2), and (3), we can obtain the relationship between the position vector $\eta$, and the velocity vector $v$, or kinematic equation of the system [15],

$$
\dot{\eta}=J(\psi) v
$$

\section{MARINE Vehicle DYNAMiC MOdel}

The marine vehicle dynamic model considered is similar to that presented in [15], the only difference being that we consider trajectories in the horizontal plane, hence the number of degrees of freedom is reduced from six to three. The considered dynamic equation is given by

$$
M \dot{v}+D(v) v+C(v) v+g(\eta)=B \tau+J^{-1}(\psi) w,
$$

where $\tau \triangleq\left[\begin{array}{ll}\tau_{1} & \tau_{2}\end{array}\right]^{\mathrm{T}}, \tau_{1}$ and $\tau_{2}$ corresponding to the surge and sway forces, respectively, $M$ is the inertia matrix, $D(v)$ is the damping matrix, $C(v)$ is the Coriolis matrix, $g(\eta)$ is the vector of restoring forces and moments, and $w \triangleq\left[\begin{array}{lll}w_{1} & w_{2} & 0\end{array}\right]^{\mathrm{T}}$ represents the force applied on the vehicle by a constant ocean current of unknown velocity and direction, expressed in the inertial frame of reference. The expressions of $M, D(v), C(v), D(v)$ and $g(\eta)$ are detailed in [15].

The matrix $B$ represents the way that our two commands are applied to the vehicle, in particular, we will consider the following case,

$$
B=\left[\begin{array}{cc}
1 & 0 \\
0 & 1 \\
0 & -L
\end{array}\right]
$$

where $L$ is the distance, along the vehicle longitudinal axis, between the vehicle's center of mass and the point where the thrust is applied. Most controllers for this underactuated problem ( [4], [6], [16]) are derived using a $B$ matrix of the form

$$
B=\left[\begin{array}{ll}
1 & 0 \\
0 & 0 \\
0 & 1
\end{array}\right]
$$


which corresponds to a vehicle equipped with two propellers and no rudder, as described in [4], [6]. Finally, in [2], $B$ is of the general form

$$
B=\left[\begin{array}{ll}
1 & 0 \\
0 & 1 \\
0 & L
\end{array}\right]
$$

which corresponds to a side thruster placed in front of the vehicle's center of gravity instead of being placed behind, which would be the case corresponding to (7). The difference between $B$ in (7) and (9) is crucial. With (9), the sway control force has the same sign as the yawing control moment, while when considering (7), they have opposite signs. This means that if the vehicle must turn right, in the case given by (9), the sway force applied to generate the desired yawing moment will move the vehicle to the right, helping the maneuver. When considering the $B$ matrix provided by (7), the sway force applied to generate the desired yawing moment will actually push the vehicle to the left, hindering the movement. This difference is reflected by the zero dynamics of the system, whose behavior is stable when considering (9), while it is not when using (7), as mentioned in [2].

\section{TRACKING ERRORS}

As mentioned in Section I, we will use a reference model. This reference model, corresponding to a virtual vehicle is chosen so that it will easily and accurately track a desired trajectory. The challenge then lies in controlling the actual vehicle so that it tracks the reference system. The dynamics of such a reference system can be arbitriraly chosen. In particular, we chose to adopt the dynamics of two uncoupled second-order oscillators. The interested reader should refer to [1] or [10] for a detailed presentation of such a choice.

\section{A. Position Errors}

The error in position in the inertial and body fixed frames of reference are respectively defined by

$$
e \triangleq \eta_{\mathrm{r}}-\eta_{\mathrm{s}}, \quad \tilde{e} \triangleq J_{\mathrm{s}}^{-1}(\psi) e,
$$

where $e$ is the error in position in the inertial frame, $\tilde{e}$ is this same error in position, but projected in the body fixed frame, $\eta_{\mathrm{s}} \triangleq\left[\begin{array}{ll}x & y\end{array}\right]^{\mathrm{T}}$ is the actual position of the vehicle, $\eta_{\mathrm{r}}$ is the position of the reference system the vehicle is tracking, and $J_{\mathrm{s}}(\psi)$ is the $(1,2) \times(1,2)$ block of $J(\psi)$.

Using (5) and (10), we obtain the following first and second time derivatives for $\tilde{e}$,

$$
\begin{aligned}
& \dot{\tilde{e}}=J_{\mathrm{s}}^{-1}(\psi) \dot{\eta}_{\mathrm{r}}-v_{\mathrm{s}}+r S \tilde{e}, \\
& \ddot{\tilde{e}}=J_{\mathrm{s}}^{-1}(\psi)\left(\ddot{\eta}_{\mathrm{r}}+r S \dot{\eta}_{\mathrm{r}}\right)-\dot{v}_{\mathrm{s}}+\dot{r} S \tilde{e}+r S \dot{\tilde{e}},
\end{aligned}
$$

where $v_{\mathrm{S}} \triangleq\left[\begin{array}{ll}u & v\end{array}\right]^{\mathrm{T}}$, and $S$ is the following skewsymmetric matrix,

$$
S \triangleq\left[\begin{array}{cc}
0 & 1 \\
-1 & 0
\end{array}\right]
$$

Note that the control action $\tau$ appears explicitly in the expression of $\ddot{\tilde{e}}(12)$ through $\dot{v}_{\mathrm{s}}$. Finally, the distance between the vehicle and its desired position is defined as

$$
e_{\mathrm{d}} \triangleq\|\tilde{e}\|=\|e\|
$$

where $\|\cdot\|$ denotes the Euclidian norm.

The time derivative of such a distance is given by

$$
\dot{e}_{\mathrm{d}}=\frac{1}{e_{\mathrm{d}}} \tilde{e}^{\mathrm{T}} \dot{\tilde{e}}
$$

\section{B. Angular Error}

The angle $\beta \in(-\pi, \pi]$ is defined as the angle between the longitudinal axis of the vehicle and the direction of the desired position. It can be computed as follows,

$$
\beta \triangleq \tan ^{-1}\left(\frac{\tilde{e}_{2}}{\tilde{e}_{1}}\right)
$$

and its time derivative is given by

$$
\dot{\beta}=\frac{1}{e_{\mathrm{d}}^{2}} \tilde{e}^{\mathrm{T}} S \dot{\tilde{e}}
$$

\section{Underactuated BACKSTEPPING DESIGN}

In this section, we derive a control law for an underactuated marine vehicle whose model is described in Section III. In particular, we show how, introducing a maximum tolerable tracking error, it is possible to overcome the zero-dynamics instability of the controlled system, and guarantee not only excellent tracking performance, but also boundedness of the uncontrolled states.

\section{A. Backstepping Technique, First Step}

We want to control the vehicle in terms of distance from the desired position $e_{\mathrm{d}}$ and angle $\beta$. Thus, we introduce this first function of the errors,

$$
V_{0}\left(e_{\mathrm{d}}, \beta\right)=e_{\mathrm{d}} \sin ^{2}\left(\frac{\beta}{2}\right)+\frac{1}{2} e_{\mathrm{d}}^{2} .
$$

Note that if $e_{\mathrm{d}}>0$, then $V_{0}\left(e_{\mathrm{d}}, \beta\right)>0, \beta \in(-\pi, \pi]$. Using (15) and (17), we can obtain the time derivative of this function,

$$
\dot{V}_{0}\left(e_{\mathrm{d}}, \beta, \dot{\tilde{e}}\right)=\left[\sin ^{2}\left(\frac{\beta}{2}\right)+e_{\mathrm{d}} \quad \frac{1}{2} \sin (\beta)\right] J^{-1}(\beta) \dot{\tilde{e}},
$$

where $J(\beta) \triangleq\left[\begin{array}{cc}\cos (\beta) & -\sin (\beta) \\ \sin (\beta) & \cos (\beta)\end{array}\right]$. We now consider $\dot{\tilde{e}}$ as our virtual command, which we would ideally choose to be equal to

$$
\dot{\tilde{e}}_{\mathrm{des}}=-e_{\mathrm{d}} J(\beta) G_{1}\left[\begin{array}{c}
\sin ^{2}\left(\frac{\beta}{2}\right)+e_{\mathrm{d}}-a \\
\frac{1}{2} \sin (\beta)
\end{array}\right],
$$

where $a>0$ is an arbitrary constant which will measure the maximum allowable tracking error. Next, in order to guarantee that $\dot{\tilde{e}}$ converges toward $\dot{\tilde{e}}_{\text {des }}$, we can introduce the following error variable

$$
\gamma \triangleq \dot{\tilde{e}}-\dot{\tilde{e}}_{\mathrm{des}}
$$

whose time derivative is given by

$$
\begin{aligned}
\dot{\gamma}=\ddot{\tilde{e}} & +\left[\begin{array}{cc}
\dot{\tilde{e}}_{1} & -\dot{\tilde{e}}_{2} \\
\dot{\tilde{e}}_{2} & \dot{\tilde{e}}_{1}
\end{array}\right] G_{1}\left[\begin{array}{c}
\sin ^{2}\left(\frac{\beta}{2}\right)+e_{\mathrm{d}}-a \\
\frac{1}{2} \sin (\beta)
\end{array}\right] \\
& +J(\beta) G_{1}\left[\begin{array}{cc}
\tilde{e}_{1}-\frac{1}{2} \sin ^{2}(\beta) & \tilde{e}_{2}+\frac{1}{4} \sin (2 \beta) \\
-\frac{1}{4} \sin (2 \beta) & \frac{1}{2} \cos ^{2}(\beta)
\end{array}\right] \dot{\tilde{e}} .
\end{aligned}
$$

Given the complexity of $\dot{\gamma}$, we choose to simplify its expression. To do so, we will use a technique derived from Dynamic Surface Control theory [14]. Instead of using $\gamma$, we will use

$$
\gamma_{\mathrm{f}} \triangleq J_{\mathrm{s}}^{-1}(\psi) \dot{\eta}_{\mathrm{r}}-v_{\mathrm{s}}+\chi
$$

where $\chi$ corresponds to $r S \tilde{e}-\dot{\tilde{e}}_{\mathrm{des}}$ passed through the following filter,

$$
T \dot{\chi}+\chi=r S \tilde{e}-\dot{\tilde{e}}_{\mathrm{des}},
$$


$T$ being a diagonal matrix whose elements are positive constants and constitute the filter time constants.

Finally, from (19), (20) and (24), we obtain that

$$
\begin{aligned}
& \dot{V}_{0}\left(e_{\mathrm{d}}, \beta, \gamma_{\mathrm{f}}\right)=\gamma_{\mathrm{f}}^{\mathrm{T}} J(\beta)\left[\begin{array}{c}
\sin ^{2}\left(\frac{\beta}{2}\right)+e_{\mathrm{d}}-a \\
\frac{1}{2} \sin (\beta)
\end{array}\right] \\
& -e_{\mathrm{d}}\left[\sin ^{2}\left(\frac{\beta}{2}\right)+e_{\mathrm{d}} \frac{1}{2} \sin (\beta)\right] G_{1}\left[\begin{array}{c}
\sin ^{2}\left(\frac{\beta}{2}\right)+e_{\mathrm{d}}-a \\
\frac{1}{2} \sin (\beta)
\end{array}\right] .
\end{aligned}
$$

\section{B. Backstepping Technique, Second Step}

We now introduce the new function

$$
V_{1}\left(e_{\mathrm{d}}, \beta, \gamma_{\mathrm{f}}\right)=e_{\mathrm{d}} \sin ^{2}\left(\frac{\beta}{2}\right)+\frac{1}{2} e_{\mathrm{d}}^{2}+\frac{1}{2} \gamma_{\mathrm{f}}^{\mathrm{T}} \gamma_{\mathrm{f}}
$$

whose time derivative is given by

$$
\begin{aligned}
& \dot{V}_{1}\left(e_{\mathrm{d}}, \beta, \gamma_{\mathrm{f}}, \tau^{*}, t\right)= \\
& -e_{\mathrm{d}}\left[\sin ^{2}\left(\frac{\beta}{2}\right)+e_{\mathrm{d}}\right. \\
& \left.\quad \frac{1}{2} \sin (\beta)\right] G_{1}\left[\begin{array}{c}
\sin ^{2}\left(\frac{\beta}{2}\right)+e_{\mathrm{d}}-a \\
\frac{1}{2} \sin (\beta)
\end{array}\right] \\
& \quad+\gamma_{\mathrm{f}}^{\mathrm{T}}\left(J(\beta)\left[\begin{array}{c}
\sin ^{2}\left(\frac{\beta}{2}\right)+e_{\mathrm{d}}-a \\
\frac{1}{2} \sin (\beta)
\end{array}\right]+\dot{\gamma}_{\mathrm{f}}\left(\tau^{*}, t\right)\right) \cdot
\end{aligned}
$$

Next, using (6), (12), and (24), we obtain

$$
\begin{aligned}
& \dot{V}_{1}\left(e_{\mathrm{d}}, \beta, \gamma_{\mathrm{f}}, \tau^{*}, t\right)=\gamma_{\mathrm{f}}^{\mathrm{T}}\left(F_{1}\left(e_{\mathrm{d}}, \beta, \chi, t\right)-G_{2} \gamma_{\mathrm{f}}\right. \\
& \left.+M_{\mathrm{s}}^{-1}\left(D_{\mathrm{s}}\left(v_{\mathrm{s}}\right)+C_{\mathrm{s}}(v)\right) v_{\mathrm{s}}-M_{\mathrm{s}}^{-1} J_{\mathrm{s}}^{-1}(\psi) w_{\mathrm{s}}-M_{\mathrm{s}}^{-1} B_{\mathrm{s}} \tau^{*}\right)
\end{aligned}
$$

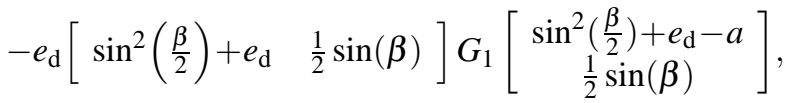

where $M_{\mathrm{s}}, D_{\mathrm{s}}\left(v_{\mathrm{s}}\right), C_{\mathrm{s}}(v)$ and $B_{\mathrm{s}}$ are the $(1,2) \times(1,2)$ blocks of $M, D(v), C(v)$ and $B$. In addition, $w_{\mathrm{s}} \triangleq\left[\begin{array}{ll}w_{1} & w_{2}\end{array}\right]^{\mathrm{T}}, G_{2}$ is a positive definite matrix, and

$$
\begin{aligned}
& F_{1}\left(e_{\mathrm{d}}, \beta, \chi, t\right) \triangleq J(\beta)\left[\begin{array}{c}
\sin ^{2}\left(\frac{\beta}{2}\right)+e_{\mathrm{d}}-a \\
\frac{1}{2} \sin (\beta)
\end{array}\right] \\
& \quad+J_{\mathrm{s}}^{-1}(\psi)\left(\ddot{\eta}_{\mathrm{r}}+r S \dot{\eta}_{\mathrm{r}}\right)+T^{-1}\left(r S \tilde{e}-\dot{\tilde{e}}_{\mathrm{des}}-\chi\right),
\end{aligned}
$$

where the explicit time dependency comes from the time varying variables $\psi$ and $\tilde{e}$. Now that our control command $\tau^{*}$ appears explicitly in the expression of $\dot{V}_{1}\left(e_{\mathrm{d}}, \beta, \gamma_{\mathrm{f}}, \tau^{*}, t\right)$, we can easily choose it in order to cancel any unwanted term. In particular, we choose

$$
\begin{aligned}
\tau^{*}=B_{\mathrm{s}}^{-1}\left[\left(D_{\mathrm{s}}\left(v_{\mathrm{s}}\right)\right.\right. & \left.+C_{\mathrm{s}}(v)\right) v_{\mathrm{s}} \\
& \left.\quad+M_{\mathrm{s}} F_{1}\left(e_{\mathrm{d}}, \beta, \chi, t\right)+J_{\mathrm{s}}^{-1}(\psi) w_{\mathrm{s}}\right] .
\end{aligned}
$$

The corresponding $\dot{V}_{1}\left(e_{\mathrm{d}}, \beta, \gamma_{\mathrm{f}}, \tau^{*}, t\right)$ is then given by

$$
\begin{aligned}
& \dot{V}_{1}\left(e_{\mathrm{d}}, \beta, \gamma_{\mathrm{f}}, \tau^{*}, t\right)=-\gamma_{\mathrm{f}}^{\mathrm{T}} G_{2} \gamma_{\mathrm{f}}
\end{aligned}
$$

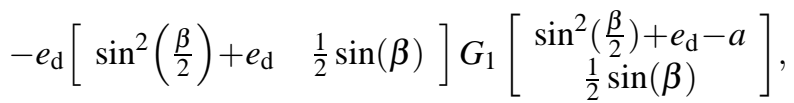

We have $V_{1}\left(e_{\mathrm{d}}, \beta, \gamma_{\mathrm{f}}\right)>0$ and $\dot{V}_{1}\left(e_{\mathrm{d}}, \beta, \gamma_{\mathrm{f}}, \tau^{*}, t\right)<0$ provided that $e_{\mathrm{d}} \geqslant a$. Using ultimate boundedness [13], we can thus guarantee that the system will enter and remain in the domain $\mathscr{D} \triangleq\left\{\beta \in(-\pi, \pi], e_{\mathrm{d}}<a\right\}$.

\section{AdAPtive Control LAW}

In this section, we modify the control law obtained in Section $\mathrm{V}$ into an adaptive one, using the framework introduced in [9], improving in this way the flexibility of the control algorithm presented in the previous section.

\section{A. Reformulating the Control Law}

First, we need all of the unknown parameters to appear linearly in our control command. We define the following notations,

$$
\begin{gathered}
\Theta_{1}^{*} \triangleq B_{\mathrm{s}}^{-1} M_{\mathrm{s}}, \Theta_{2}^{*} \triangleq B_{\mathrm{s}}^{-1} D_{\mathrm{ls}}, \Theta_{3}^{*} \triangleq B_{\mathrm{s}}^{-1} D_{\mathrm{qs}} \\
\Theta_{4}^{*} \triangleq B_{\mathrm{s}}^{-1}\left[\begin{array}{cc}
m_{2} & 0 \\
0 & m_{1}
\end{array}\right], \Theta_{5}^{*} \triangleq B_{\mathrm{s}}^{-1}\left[\begin{array}{cc}
w_{1} & w_{2} \\
w_{2} & -w_{1}
\end{array}\right] \\
F_{2}\left(v_{\mathrm{s}}\right) \triangleq v_{\mathrm{s}}, F_{3}\left(v_{\mathrm{s}}\right) \triangleq\left[\begin{array}{cc}
\|u\| & 0 \\
0 & \|v\|
\end{array}\right] v_{\mathrm{s}}, F_{4}(v) \triangleq r S^{\mathrm{T}} v_{\mathrm{s}} \\
F_{5}(\psi) \triangleq\left[\begin{array}{c}
\cos \psi \\
\sin \psi
\end{array}\right]
\end{gathered}
$$

where $D_{\mathrm{ls}}$ and $D_{\mathrm{qs}}$ are the coefficients of the linear and quadratic part of $D_{\mathrm{s}}$, respectively, and we rewrite our command $\tau^{*}$ as

$$
\begin{aligned}
\tau^{*}=\Theta_{1}^{*} F_{1}\left(e_{\mathrm{d}}, \beta, \chi, t\right) & +\sum_{i=2}^{3} \Theta_{i}^{*} F_{i}\left(v_{\mathrm{s}}\right) \\
& +\Theta_{4}^{*} F_{4}(v)+\Theta_{5}^{*} F_{5}(\psi) .
\end{aligned}
$$

Next, considering that we do not have the exact values of $\Theta_{i}^{*}, i=1,2,3,4,5$, we are going to use an estimate $\Theta$ for each $\Theta^{*}$. The command we will use is thus

$$
\begin{aligned}
\tau=\Theta_{1} F_{1}\left(e_{\mathrm{d}}, \beta, \chi, t\right)+ & \sum_{i=2}^{3} \Theta_{i} F_{i}\left(v_{\mathrm{s}}\right) \\
& +\Theta_{4} F_{4}(v)+\Theta_{5} F_{5}(\psi) .
\end{aligned}
$$

The error between the estimate $\Theta$ and the actual value $\Theta^{*}$ will by referred to as $\tilde{\Theta}$,

$$
\tilde{\Theta} \triangleq \Theta-\Theta^{*} \text {. }
$$

\section{B. New Function of the Error}

By using the control law (34) instead of (33), we introduce new terms in $\dot{V}_{1}$. In order to compensate for those terms, we introduce the new function of the error

$$
\begin{aligned}
& V_{2}\left(e_{\mathrm{d}}, \beta, \gamma_{\mathrm{f}}, t\right)=e_{\mathrm{d}} \sin ^{2}\left(\frac{\beta}{2}\right)+\frac{1}{2} e_{\mathrm{d}}^{2}+\frac{1}{2} \gamma_{\mathrm{f}}^{\mathrm{T}} \gamma_{\mathrm{f}} \\
& +\sum_{i=1}^{5} \frac{1}{2} \operatorname{tr}\left(\mathrm{M}_{\mathrm{s}}^{-1} \mathrm{~B}_{\mathrm{s}} \tilde{\Theta}_{i} \Gamma_{i}^{-1} \tilde{\Theta}_{i}^{\mathrm{T}}\right)
\end{aligned}
$$

where $\operatorname{tr}(\cdot)$ is the trace operator, $\Gamma_{i}, i=1,2,3,4,5$ are positive design constants. The corresponding time derivative is given 
by

$$
\begin{aligned}
& \dot{V}_{2}\left(e_{\mathrm{d}}, \beta, \gamma_{\mathrm{f}}, \tau, t\right)=-\gamma_{\mathrm{f}}^{\mathrm{T}} G_{2} \gamma_{\mathrm{f}} \\
& -e_{\mathrm{d}}\left[\sin ^{2}\left(\frac{\beta}{2}\right)+e_{\mathrm{d}} \quad \frac{1}{2} \sin (\beta)\right] G_{1}\left[\begin{array}{c}
\sin ^{2}\left(\frac{\beta}{2}\right)+e_{\mathrm{d}}-a \\
\frac{1}{2} \sin (\beta)
\end{array}\right] \\
& -\operatorname{tr}\left(\mathrm{M}_{\mathrm{s}}^{-1} \mathrm{~B}_{\mathrm{s}}^{-1} \tilde{\Theta}_{1} \mathrm{~F}_{1}\left(\mathrm{e}_{\mathrm{d}}, \beta, \mathrm{t}\right) \gamma_{\mathrm{f}}^{\mathrm{T}}-\mathrm{M}_{\mathrm{s}}^{-1} \mathrm{~B}_{\mathrm{s}} \tilde{\Theta}_{1} \Gamma_{1}^{-1} \dot{\Theta}_{1}^{\mathrm{T}}\right) \\
& -\sum_{i=2}^{3} \operatorname{tr}\left(\mathrm{M}_{\mathrm{s}}^{-1} \mathrm{~B}_{\mathrm{s}} \tilde{\Theta}_{i} \mathrm{~F}_{i}\left(v_{\mathrm{s}}\right) \gamma_{\mathrm{f}}^{\mathrm{T}}-\mathrm{M}_{\mathrm{s}}^{-1} \mathrm{~B}_{\mathrm{s}} \tilde{\Theta}_{i} \Gamma_{i}^{-1} \dot{\Theta}_{i}^{\mathrm{T}}\right) \\
& -\operatorname{tr}\left(\mathrm{M}_{\mathrm{s}}^{-1} \mathrm{~B}_{\mathrm{s}} \tilde{\Theta}_{4} \mathrm{~F}_{4}(v) \gamma_{\mathrm{f}}^{\mathrm{T}}-\mathrm{M}_{\mathrm{s}}^{-1} \mathrm{~B}_{\mathrm{s}} \tilde{\Theta}_{4} \Gamma_{4}^{-1} \dot{\Theta}_{4}^{\mathrm{T}}\right) \\
& -\operatorname{tr}\left(\mathrm{M}_{\mathrm{s}}^{-1} \mathrm{~B}_{\mathrm{s}} \tilde{\Theta}_{5} \mathrm{~F}_{5}(\psi) \gamma_{\mathrm{f}}^{\mathrm{T}}-\mathrm{M}_{\mathrm{s}}^{-1} \mathrm{~B}_{\mathrm{s}} \tilde{\Theta}_{5} \Gamma_{5}^{-1} \dot{\Theta}_{5}^{\mathrm{T}}\right)
\end{aligned}
$$

Finally, by choosing the following update laws for $\dot{\Theta}_{i}, i=$ $1,2,3,4,5$,

$$
\begin{aligned}
\dot{\Theta}_{1} & =\Gamma_{1} \gamma_{\mathrm{f}} F_{1}^{\mathrm{T}}\left(e_{\mathrm{d}}, \beta, t\right), \\
\dot{\Theta}_{i} & =\Gamma_{i} \gamma_{\mathrm{f}} F_{i}^{\mathrm{T}}\left(v_{\mathrm{s}}\right), \quad i=2,3 \\
\dot{\Theta}_{4} & =\Gamma_{4} \gamma_{\mathrm{f}} F_{4}^{\mathrm{T}}(v), \\
\dot{\Theta}_{5} & =\Gamma_{5} \gamma_{\mathrm{f}} F_{5}^{\mathrm{T}}(\psi)
\end{aligned}
$$

the expression of $\dot{V}_{2}$ becomes

$$
\begin{aligned}
& \dot{V}_{2}\left(e_{\mathrm{d}}, \beta, \gamma_{\mathrm{f}}, \tau, t\right)=-\gamma_{\mathrm{f}}^{\mathrm{T}} G_{2} \gamma_{\mathrm{f}}
\end{aligned}
$$

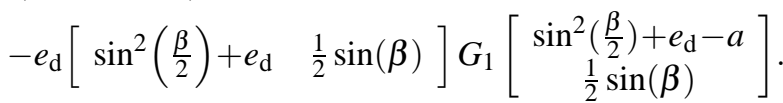

Having $V_{2}\left(e_{\mathrm{d}}, \beta, \gamma_{\mathrm{f}}, t\right)>0$ and $\dot{V}_{2}\left(e_{\mathrm{d}}, \beta, \gamma_{\mathrm{f}}, \tau, t\right)<0$ if $e_{\mathrm{d}} \geqslant a$, we can guarantee, using ultimate boundedness theory, that the system will enter and remain in the domain $\mathscr{D}$ previously defined.

\section{Simulation RESUlts}

\section{A. Circular Trajectory}

The first maneuver we will attempt is a counterclockwise circle of radius $10 \mathrm{~m}$ at a velocity of $1 \mathrm{~m} / \mathrm{s}$, with the following initial conditions,

$$
\eta=\left[\begin{array}{lll}
0 & 0 & 0
\end{array}\right]^{\mathrm{T}}, \quad \boldsymbol{v}=\left[\begin{array}{lll}
0 & 0 & 0
\end{array}\right]^{\mathrm{T}} .
$$

The reference model initial conditions are

$$
\eta_{\mathrm{r}}(0)=\left[\begin{array}{ll}
0.6 & 0
\end{array}\right]^{\mathrm{T}} \text {. }
$$

The reference system is modelled after a pair of second order oscillators and is detailed in [1]. The natural frequency and damping matrices of the reference system are set at $0.2 \mathrm{I}_{2}$ and $0.9 \mathrm{I}_{2}$, respectively, where $\mathrm{I}_{2}$ is the $2 \times 2$ identity matrix.

The initials conditions chosen for the $\Theta$ estimates are

$$
\begin{gathered}
\Theta_{1}=\left[\begin{array}{cc}
15 & 0 \\
0 & 50
\end{array}\right], \\
\Theta_{2}=\Theta_{3}=\left[\begin{array}{cc}
-5 & 0 \\
0 & 10
\end{array}\right], \quad \Theta_{4}=\left[\begin{array}{cc}
40 & 0 \\
0 & 10
\end{array}\right],
\end{gathered}
$$

In this first maneuver, we will assume there is no ocean current to perturb the motion of the vehicle. Thus, the initial value for $\Theta_{5}$ will be zero, and we will set $\Gamma_{5}=0$.

Furthermore, $\Gamma_{i}=I_{2}, i=1,2,3,4, a=0.6$, and $G_{1}=G_{2}=$ $I_{2}$. The dynamic model of the vehicle corresponds to the
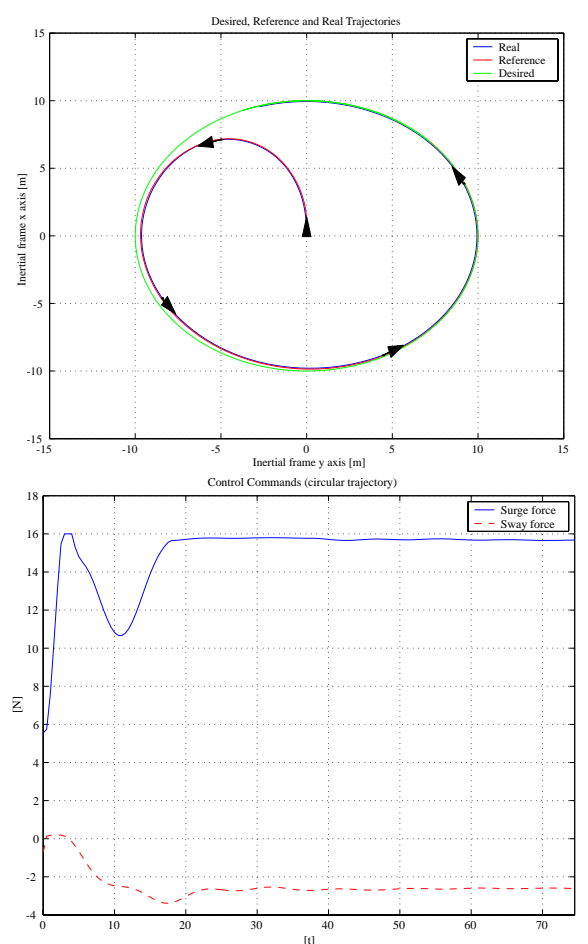

Fig. 1. Circular trajectory and corresponding control efforts

Silent Quick Unmanned Intelligent Diver [1]. The values for $M, D_{\mathrm{l}}$ and $D_{\mathrm{q}}$ are given in [1].

Finally, the initial position of the desired trajectory is

$$
\eta_{\mathrm{ds}}(0)=\left[\begin{array}{ll}
7.0711 & 7.0711
\end{array}\right]^{\mathrm{T}} .
$$

As shown in Figure 1, the tracking performances are excellent, and the vehicle is moving in a coherent fashion (the arrows show the orientation of the vehicle).

We used the framework introduced in [9] to enforce command input amplitude saturation, and keep the control efforts at a reasonable level, as shown in Figure 1. Finally, the $\Theta$ estimates converge quickly and remain stable (Figure 2).

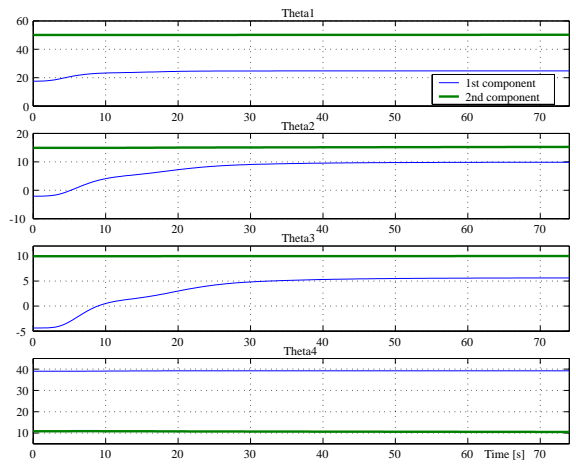

Fig. 2. Relevant $\Theta$ gains (circular maneuver without perturbation) 


\section{B. Robustness to External Perturbations}

We will now consider the motion of the vehicle when it is perturbed by a steady current. The current will be modelled as a force, constant in the inertial frame of reference, similarly to the approach presented in [2]. The value of the current vector is $w \triangleq\left[\begin{array}{lll}0.2 & 0.2 & 0\end{array}\right]^{\mathrm{T}}$. Furthermore, we choose $\Theta_{5}=10\left[\begin{array}{cc}1 & 1 \\ 1 & -1\end{array}\right]$ and $\Gamma_{5}=1$. The desired trajectory, the dynamic model constants and initial conditions are otherwise similar to those of the first maneuver.
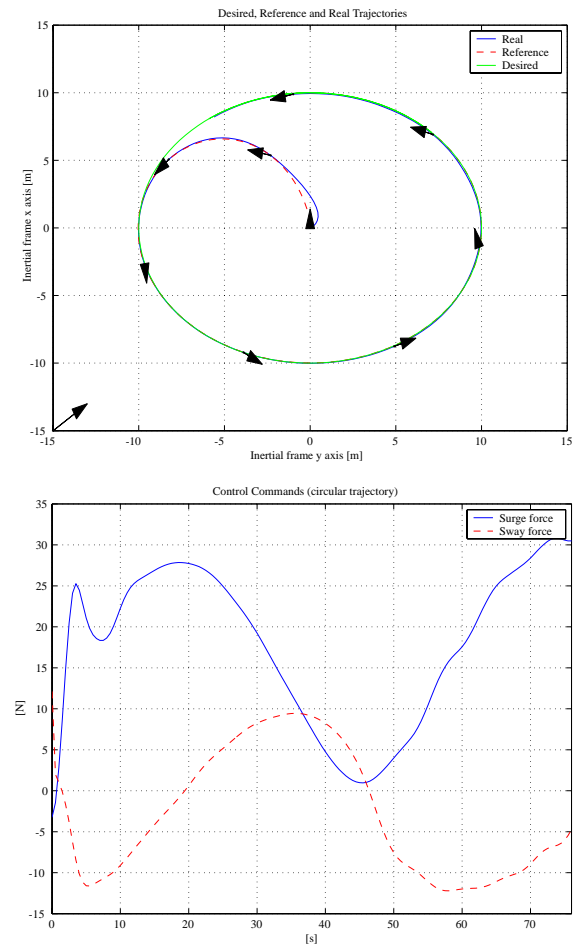

Fig. 3. Circular trajectory with external perturbations and corresponding control efforts

The bottom left corner arrow displays the direction of the current. The tracking properties are still good, although the vehicle slightly drifts in the direction of the current. It can be noticed in Figure 3 that the vehicle orients itself in order to minimize the influence of the current. To get the vehicle's behavior to be noticeably influenced by this external perturbation, we had to use large numerical values of the force modelling the current. Choosing such values for the external perturbation, we had to remove the saturation constraints for the vehicle to be able to cope with the current (Figure 3).

\section{CONCLUSION}

In this paper, a backstepping approach and a technique derived from dynamic surface control theory were used to derive a nonlinear tracking control law for underactuated nonminimum phase marine vehicles. The resulting control law can be applied to solve the trajectory tracking problem for any marine vehicle using a propeller and a rudder, or a vectored thruster. The flexibility of the result has been increased by introducing update laws for the estimates of the vehicle's model unknown parameters. It can furthermore handle unknown, constant external perturbations. Computer simulations illustrate the excellent performances of the control algorithm and its robustness to external perturbations.

\section{REFERENCES}

[1] Y. Morel, "Design of an adaptive nonlinear controller for an autonomous underwater vehicle equipped with a vectored thruster," Master Thesis, Florida Atlantic University, 2002.

[2] T. Fossen, J.-M. Godhavn, S. Berge, and K.-P. Lindegaard, "Nonlinear control of underactuated ships with forward speed compensation," in Proc. IFAC NOLCOS98, vol. 1, (Enschede, Netherlands), pp. 121-126, 1998.

[3] G. Toussaint, T. Basar, and F. Bullo, "Tracking for nonlinear underactuated surface vessels with generalized forces," 2000.

[4] G. Toussaint, T. Basar, and F. Bullo, " $h^{\infty}$-optimal tracking control techniques for nonlinear underactuated systems," in Proc. 39th IEEE Conf. Dec. Contr., vol. 3, (Sydney, Australia), pp. 2078-2083, 2000.

[5] P. M. M. Encarnacão, "Nonlinear path following control systems for ocean vehicles," PHD Thesis, Instituto Superior Technico, 2002.

[6] J.-M. Godhavn, "Nonlinear control of underactuated surface vessels," in Proc. 35th IEEE Conf. Dec. Contr., vol. 1, (Kobe, Japan), pp. 975-980, 1996.

[7] A. Aguiar and A. Pascoal, "Regulation of a nonholonomic autonomous underwater vehicle with parametric modeling uncertainty using lyapunov functions," in Proc. 40th IEEE Conf. Dec. Contr., (Orlando, FL), pp. 4178-4183, 2001.

[8] S. F. O.-E. Fjellstad and O. Egeland, "Adaptive control of rovs with actuator dynamics and saturation," Modeling, Identification and Control, vol. 13, pp. 175-188, 1992.

[9] A. Leonessa, W. Haddad, and T. Hayakawa, "Adaptive tracking for nonlinear systems with control constraints," in Proc. Amer. Contr. Conf., vol. 2, (Arlington, VA), pp. 1292-1297, 2001.

[10] Y. Morel and A. Leonessa, "Adaptive nonlinear heading control of an underactuated nonminimum phase model of a marine vehicle," in Proc. 4th Asian Contr. Conf., (Singapore), pp. 117-122, 2002.

[11] J. Slotine and W. Li, Applied Nonlinear Control. Englewood Cliffs, New Jersey: Prentice Hall, 1991.

[12] M. Krstić, I. Kanellakopoullos, and P. Kokotović, Nonlinear and Adaptive Control Design. New York, NY: John Wiley and Sons, Inc, 1995.

[13] H. K. Khalil, Nonlinear Systems. Prentice-Hall, Upper Saddle River, NJ, 1996.

[14] P.-P. Y. D. Swaroop, J.-K. Hedrick and J.-C. Gerdes, "Dynamic surface control for a class of nonlinear systems," IEEE Trans. Autom. Contr., vol. 45, pp. 18931899, 2000.

[15] T. Fossen, Guidance and Control of Ocean Vehicles. Baffins Lane, England: John Wiley and Sons, Inc, 1994.

[16] K. Pettersen and H. Nijmeijer, "Tracking control of an underactuated surface vessel," in Proc. 37th IEEE Conf. Dec. Contr., vol. 4, (Tampa, FL), pp. 4561-4566, 1998. 